\title{
Emotions in reading: Dissociation of happiness and positivity
}

\author{
Benny B. Briesemeister • Lars Kuchinke • Arthur M. Jacobs • Mario Braun
}

Published online: 15 November 2014

(C) Psychonomic Society, Inc. 2014

\begin{abstract}
The hierarchical emotion model proposed by Panksepp (1998) predicts that affective processing will rely on three functionally and neuroanatomically distinct levels, engaging subcortical networks (primary level), the limbic system (secondary level), and the neocortex (tertiary level). In the present fMRI study, we manipulated happiness and positivity, which are assumed to rely on secondary- and tertiary-level processes, respectively, to test these assumptions in a word recognition task. In accordance with the model predictions, evidence for a double dissociation was found in the brain activation patterns: Secondary-level processes engaged parts of the limbic system — specifically, the right
\end{abstract}

Electronic supplementary material The online version of this article (doi:10.3758/s13415-014-0327-2) contains supplementary material, which is available to authorized users.

B. B. Briesemeister · A. M. Jacobs $\cdot$ M. Braun

Allgemeine und Neurokognitive Psychologie, Freie Universität

Berlin, Berlin, Germany

B. B. Briesemeister

Center for Applied Neuroscience, Freie Universität Berlin, Berlin, Germany

\section{Kuchinke}

Experimentelle Psychologie und Methodenlehre, Ruhr-Universität Bochum, Bochum, Germany

\section{A. M. Jacobs}

Dahlem Institute for Neuroimaging of Emotion (D.I.N.E.), Berlin, Germany

\section{Braun}

Fachbereich Psychologie \& Center for Neurocognitive Research, Universität Salzburg, Salzburg, Austria

\section{B. B. Briesemeister $(\square)$}

Department of Psychology, Freie Universität Berlin,

Habelschwerdter Allee 45, 14195 Berlin, Germany

e-mail: benny.briesemeister@fu-berlin.de hemispheric amygdala. Tertiary-level processes, in contrast, relied predominantly on frontal neocortical structures such as the left inferior frontal and medial frontal gyri. These results are interpreted as support for Panksepp's (1998) model and as an indicator of a semantic foundation of affective dimensions.

Keywords Discrete emotions - Affective dimensions · Lexical decision $\cdot$ Hierarchical affective processing

Even though considerable recent research on human affective processing has relied on neuroimaging and electrophysiological evidence (Fritsch \& Kuchinke, 2013; Satpute et al., 2013; Wilson-Mendenhall, Barrett, \& Barsalou, 2013), most theories do not explicitly specify how and where exactly emotions are represented within the human brain (Lindquist, Wager, Kober, Bliss-Moreau, \& Barrett, 2012). One of only a few notable exceptions is the hierarchical emotion theory proposed by Panksepp (1998, 2012), which generalizes evidence derived from electrical stimulation studies in animals to all mammalian species, and thus allows for neuroanatomically precise predictions. For example, Panksepp (2007) was among the first to suggest that the traditionally opposing concepts of discrete emotions, which describe emotions as a limited set of functionally distinct categories, and affective dimensions, which describe emotions as fluctuating states within two- or higher-dimensional spaces, are not alternatives, but refer to different affective processing levels within a neuroanatomically distinguishable threelevel hierarchy. At the primary process level, seven distinct emotional systems can be inferred from animal research. SEEKING, RAGE, LUST, PLAY, FEAR, PANIC, and CARE are considered to be hardwired, unconditioned emotion processes that originate in emotion-specific subcortical circuits within the periaqueductal gray area (PAG) and the lower limbic system meant to provide fixed-action patterns that "allow organisms to face key environmental challenges with little need for individual 
learning" (see Panksepp, 2012, p. 7). Satpute et al. (2013) recently demonstrated that the PAG, a core structure within the FEAR network known to be responsible for freezing and flight behavior in animals, is also involved in the processing of highly aversive images in humans. However, it is very difficult to noninvasively study unconditioned primary-process-level emotions in humans, given that the involved structures are often very small $(\sim 10 \times 6 \times 3 \mathrm{~mm}$, or approximately six standard voxels, in the case of the PAG) and are adjacent to the cerebral aqueduct, which can cause magnetic inhomogeneities (Panksepp, 2012; Satpute et al., 2013). This is why researchers typically rely on secondary-process-level conditioned affective processes. Affective conditioning (e.g., fear conditioning; LeDoux, 2000) relies on the pairing of unconditioned affective stimuli (primary-process-level emotions) and the resulting unconditioned response to previously neutral stimuli. In the case of word recognition, for example, Fritsch and Kuchinke (2013) demonstrated that the pairing of meaningless letter strings (pseudowords) with highly aversive images results in a conditioned event-related potential (ERP) effect on the N1 component ( $\sim 100 \mathrm{~ms})$ that strongly resembles early ERP effects known from implicit affective word processing (Citron, 2012; see Bayer, Sommer, \& Schacht, 2012, for a more detailed discussion on early ERP effects and conditioning). According to Panksepp, tertiary-process-level emotions, finally, rely on phylogenetically younger neocortical prefrontal brain structures and reflect higher-order categorization, reorganization, and appraisal processes. Discrete emotions are shaped by sociocultural demands and clustered "into constellations of positive and negative affect" (see Panksepp, 2006, p. 22). Following this hierarchical theory of emotion, affective dimensions are derived from more basal discrete emotions, which requires more complex empirical tests.

Wilson-Mendenhall et al. (2013) asked their participants to immerse themselves in written scenarios meant to induce feelings of fear, happiness, and sadness, and then to judge the resulting affective experience while the associated brain activity was recorded with fMRI. The scenarios were constructed to elicit both positive and negative feelings for each discrete emotion category; that is, they were "describing the pleasant fear of thrill seeking, the pleasant sadness of nostalgia, and the unpleasant happiness of unshared success " (WilsonMendenhall et al., 2013, p. 948), as well as prototypical happiness, fear, and sadness scenarios. Discrete emotions and affective dimensions were thus manipulated within one single experiment. The analyses revealed that participants' subjective valence judgments were correlated with activity in the orbitofrontal cortex (see also Lewis, Critchley, Rotshtein, \& Dolan, 2007), both within and across discrete emotion categories, whereas arousal judgments correlated with activity within the left amygdala. Wilson-Mendenhall et al. discussed these findings as evidence for a two-dimensional core affect - that is, valence and arousal-underlying all affective experiences.
Following the assumptions of the hierarchical emotion theory, however, valence judgments that correlate with orbitofrontal activations rely on higher-order evaluations, and thus on processing at the cortico-frontal tertiary process level (Panksepp, 2007). The amygdala, in contrast, is assumed to be a primaryprocess-level structure, but with amygdala subregions discussed as being involved in different emotion systems (see Table 1 in Panksepp, 2001; also Wilson-Mendenhall et al., 2013). Of note is that the amygdala is also often found active during fear conditioning (e.g., Duvarci, Popa, \& Paré, 2011; Maren, Phan, \& Liberzon, 2013; Phillips \& LeDoux, 1992); thus, it is also likely to be active in secondary-process-level conditioned responses.

Recent evidence supporting the hierarchical emotion theory has come from an ERP study in which participants were presented with affective words in a lexical decision task (LDT; Briesemeister, Kuchinke, \& Jacobs, 2014). Word lists "high" or "low" in normative discrete emotion measures of happiness and "neutral" or "positive" on the valence dimension (positivity) were orthogonally manipulated. The underlying assumption was that words rated as being highly related to happiness would activate conditioned emotion networks to a stronger extent than would words rated as being weakly related to happiness, whereas positive words would activate higher-order appraisal networks, as compared to neutral words. Such a manipulation would allow us to study the temporal signature of secondary (happiness) and tertiary (positivity) process-level brain responses. The happiness manipulation affected the very early N1 component, around $100 \mathrm{~ms}$ after stimulus onset, which is known to be sensitive to affective conditioning (Fritsch \& Kuchinke, 2013). The positivity manipulation, in contrast, affected the late $\mathrm{N} 400$ component and the late positive complex (LPC), both of which are discussed as reflecting explicit affective evaluation following word identification (Citron, 2012). No interaction between positivity and happiness was observed. These brain electrical data thus strongly support Panksepp's theory, given that happiness words, assumed to primarily modulate activity within the secondary process level, affect an ERP component that precedes those affected by positivity words, which are assumed to primarily modulate activity within the tertiary process level. Moreover, the processes that are discussed as underlying these specific ERP components - that is, initial attentional resource allocation (N1), prolonged lexicosemantic processing (N400), and especially higher-order evaluation (LPC; see Citron, 2012) - are also well in line with the model, given that Panksepp (2012) located affective-driven cognitive processes (N400, LPC) at the tertiary process level.

An alternative, complementary approach for differentiating between the secondary and tertiary process levels would be to focus on the involved brain structures rather than on the temporal dynamics, in particular since Panksepp (1998, 2012) makes very precise neuroanatomic predictions. This was the goal of the present study, which we meant to replicate and extend the results of Briesemeister et al. (2014) 
Table 1 Descriptive statistics for the stimulus set, along with the behavioral responses

\begin{tabular}{|c|c|c|c|c|c|c|}
\hline & lowHAP + NEU & lowHAP + POS & highHAP + NEU & highHAP + POS & $F$ Value & $p$ Value \\
\hline Happiness & $2.3(0.2)$ & $2.4(0.1)$ & $2.9(0.2)$ & $2.9(0.2)$ & 74.760 & $<.001$ \\
\hline Positivity & $0.5(0.2)$ & $1.3(0.2)$ & $0.5(0.2)$ & $1.4(0.2)$ & 142.052 & $<.001$ \\
\hline Log frequency & $2.3(2.1)$ & $1.8(1.9)$ & $1.8(1.4)$ & $2.2(1.7)$ & 0.747 & .526 \\
\hline Letters & $6.1(1.4)$ & $6.2(1.2)$ & $6.1(1.4)$ & $5.9(1.2)$ & 0.358 & .783 \\
\hline Syllables & $2.0(0.7)$ & $2.1(0.5)$ & $2.1(0.8)$ & $2.0(0.6)$ & 0.287 & .834 \\
\hline Phonemes & $5.5(1.3)$ & $5.7(1.3)$ & $5.4(1.4)$ & $5.3(1.2)$ & 0.721 & .541 \\
\hline Arousal & $2.4(0.3)$ & $2.5(0.6)$ & $2.6(0.6)$ & $2.5(0.8)$ & 0.572 & .634 \\
\hline Imageability & $4.7(1.3)$ & $4.6(1.5)$ & $4.9(1.4)$ & $4.9(1.5)$ & 0.256 & .857 \\
\hline Bigram frequency & $182,953(129,198)$ & $224,222(166,890)$ & $196,547(158,145)$ & $164,361(133,714)$ & 0.871 & .458 \\
\hline Ortho. neighbors $(N)$ & $1.7(1.9)$ & $2.2(3.4)$ & $1.2(2.2)$ & $1.2(2.2)$ & 0.915 & .436 \\
\hline Frequency of $N$ & $116.8(317.7)$ & $210.6(568.6)$ & $161.2(463.2)$ & $127.9(524.7)$ & 0.233 & .873 \\
\hline Higher frequent $N(\mathrm{HN})$ & $0.5(1.4)$ & $0.6(1.2)$ & $0.9(1.6)$ & $0.4(1.3)$ & 0.537 & .658 \\
\hline Frequency of $\mathrm{HN}$ & $82.4(295.0)$ & $192.6(533.7)$ & $157.7(456.2)$ & $115.4(505.7)$ & 0.333 & .801 \\
\hline Response times & $719(64)$ & $765(76)$ & $719(63)$ & $741(61)$ & & \\
\hline \multirow[t]{2}{*}{ Error rates } & $1.2(1.5)$ & $1.6(1.4)$ & $0.9(1.0)$ & $1.3(1.2)$ & & \\
\hline & lowHAP & highHAP & NEU & POS & & \\
\hline Response times & $742(85)$ & $730(74)$ & $719(61)$ & $753(65)$ & & \\
\hline Error rates & $1.4(1.3)$ & $1.1(0.9)$ & $1(0.8)$ & $1.4(0.9)$ & & \\
\hline
\end{tabular}

The rows show stimulus characteristics, including the experimental and control variables of the stimulus set, as well as mean response times and error rates

using functional magnetic resonance imaging (fMRI) instead of ERPs. On the basis of Panksepp's hierarchical emotion theory, we derived the following hypotheses:

Recent studies have suggested that the processing of single affective words relies on emotion networks in the brain, such as the anterior and posterior cingulate cortex, the medial temporal lobe including hippocampus and parahippocampal gyrus, the amygdala, and the orbitofrontal cortex (Citron, 2012; Herbert et al., 2009; Kuchinke et al., 2005; Lewis et al., 2007; Nakic, Smith, Busis, Vythilingam, \& Blair, 2006; Ponz et al., 2013; Schlochtermeier et al., 2013). Work by Ponz et al. (2013), for example, has shown that the insular cortex, which is strongly related to disgust processing (Wicker et al., 2003), is also involved in reading disgust-related words. The authors interpreted this finding in terms of neural reuse (M. L. Anderson, 2010; Herbert et al., 2009), suggesting that phylogenetically younger processes such as reading rely at least partially on already-existing old emotion processing regions - for example, the limbic system (see also Bohrn, Altmann, Lubrich, Menninghaus, \& Jacobs, 2013; Jacobs, $2011,2014)$. Words strongly related to a given emotion are thus assumed to activate a given affective program more strongly than words judged as being weakly related to that emotion, or than neutral words (Briesemeister, Kuchinke, \& Jacobs, 2011a; Jacobs \& Schrott, 2013). An fMRI replication of Briesemeister et al.'s (2014) ERP results was therefore expected to reveal distinct activations within emotionprocessing networks: Secondary-process-level emotions should rely on limbic brain structures, whereas tertiaryprocess-level emotions should engage prefrontal brain regions. Previous work has already shown that discrete emotion words explain specific variances during lexical processing, even when the stimulus material is controlled with respect to affective dimensions (Briesemeister, Kuchinke, \& Jacobs, 2011b, 2014; Silva, Montant, Ponz, \& Ziegler, 2012; Weigand et al., 2013), indicating separable underlying processes. Assuming that discrete emotion words indeed induce conditioned responses that mainly access the secondary process level, a happiness manipulation like the one described in Briesemeister et al. (2014) should, according to Panksepp (2001, Table 2), relate to the PLAY system. The primaryprocess-level circuitry underlying PLAY consists of the parafascicular and posterior thalamic nuclei, the somatosensory cortex, the hippocampus, and the dorsal PAG (Panksepp, 1998, 2001), but as we already mentioned, Panksepp (2012) and Satpute et al. (2013) agree that the primary process level is difficult to access using fMRI. Moreover, it is unknown to what extent the primary process level is accessed by conditioned affective stimuli-for example, emotional words. The secondary process level, in contrast, which can be accessed during word recognition tasks and the use of (conditioned) discrete emotion words, is not necessarily specific for a single primary-process-level emotion, but is predicted to rely on activation of the cerebellum, the temporal lobe, the amygdala, the lateral hypothalamus, and the cingulate cortex in the context of PLAY (see Panksepp, 1998, p. 291). Thus, greater 
Table 2 Inference statistics for the behavioral and fMRI data

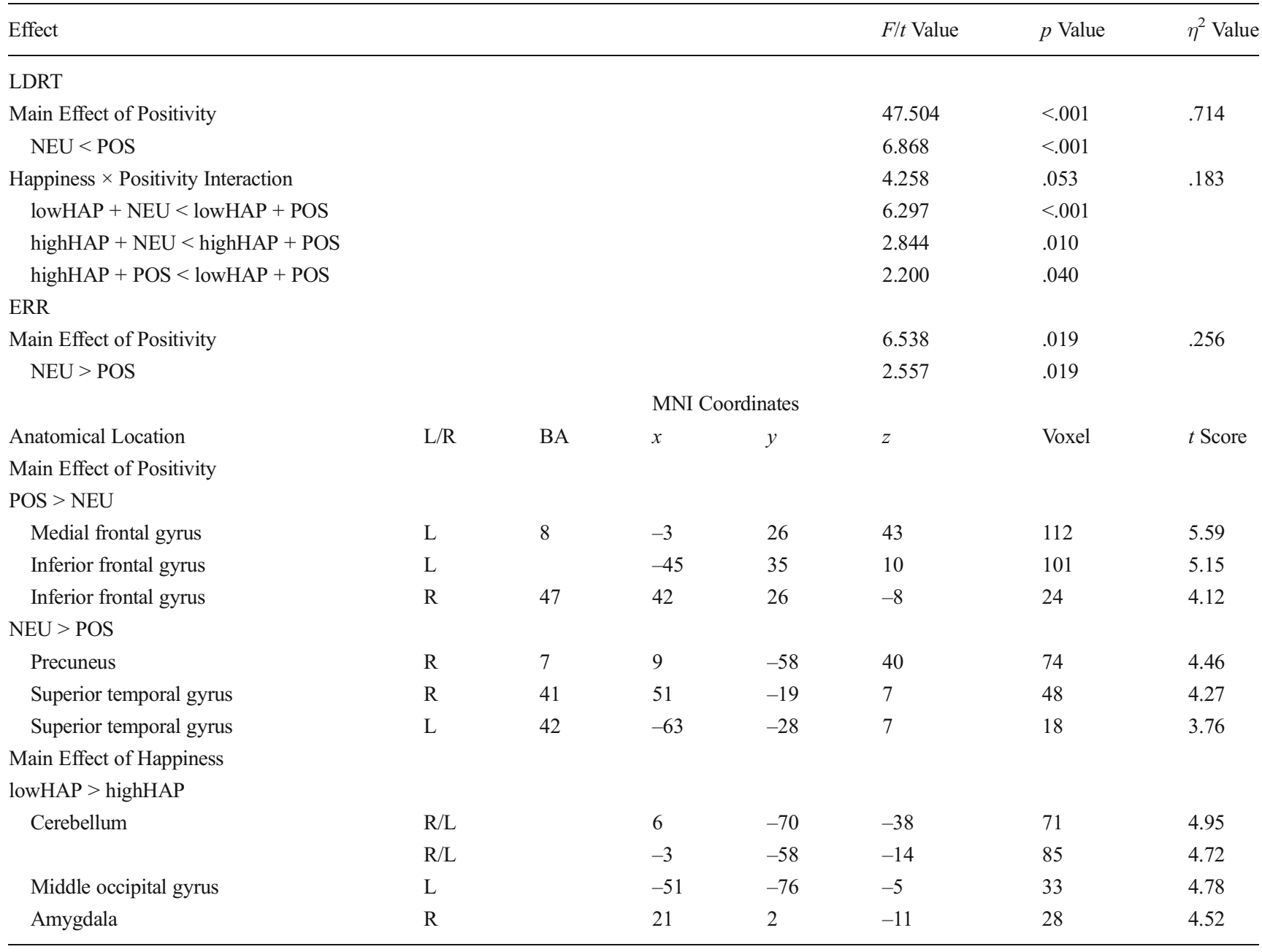

Anatomical locations for significant main effects of positivity and happiness are at $p>.001$, corrected for cluster size $(>17)$

activity within this PLAY network was expected for words high in happiness than for words low in happiness. With regard to the processing of valence along the positivity dimension (e.g., Briesemeister, Kuchinke, \& Jacobs, 2012), which is expected to primarily access the tertiary process level within the hierarchical emotion theory, greater activation with increasing positivity was expected to be observed in higherorder neocortical brain regions such as the orbitofrontal and medial frontal cortices (Lewis et al., 2007; Panksepp, 2007; Schlochtermeier et al., 2013).

\section{Method}

Participants

A total of 20 right-handed native German speakers (12 female, eight male; mean age $=23$ years, $S D=2.7$, range 19-35) were recruited at the Freie Universität Berlin. They had normal or corrected-to-normal vision and reported no known neurological condition or psychiatric illness. Prior to the experiment, participants gave written informed consent in accordance with the guidelines set by the Charite Ethics Committee at Freie Universität Berlin. The participants were compensated $€ 15$ for their participation.

Stimuli

Following Briesemeister et al. (2014), 120 German four- to eight-letter nouns and an equal number of nonwords were presented in a 2 (happiness) $\times 2$ (positivity) within-subjects design with 30 items per cell. In order to investigate the specific contributions of discrete emotions and affective dimensions to affective word recognition, the present study relied on two published affective norm databases, the Berlin Affective Word List-Reloaded (BAWL-R; Võ et al., 2009) and its discrete-emotion extension, the Discrete Emotion Norms for Nouns BAWL (DENN-BAWL; Briesemeister 
et al., 2011a). The BAWL-R provides rating-based affective norms for affective valence (7-point Likert scale, ranging from negative [-3] to positive [3]) and arousal (5-point Likert scale, ranging from low [1] to high arousing [5]) for almost 3,000 German words. Given that positivity judgments and BAWLR's valence ratings are highly correlated (Briesemeister, Hofmann, Kuchinke, \& Jacobs, 2012), words with BAWL-R scores between -0.7 and 0.7 were defined as being neutral (NEU), and words with valence scores above 1 were defined as being positive (POS), thus covering the entire positivity spectrum. The DENN-BAWL norms were used to additionally classify words as being either strongly or not strongly related to happiness, with low-happiness words (lowHAP) having DENN-BAWL happiness scores below 2.6 and highhappiness words (highHAP) having scores above 2.6. The rationale behind that was that the DENN-BAWL norms indicate the extent to which a single word is related to one of five specific discrete-emotion categories, with high scores indicating a strong relation, and thus intense activation of the underlying affective processes. The stimuli were selected so as to aim at a maximum manipulation of both variables. The resulting four orthogonal conditions (lowHAP + NEU: e.g., "HUHN," Engl. "CHICKEN; lowHAP + POS: e.g., "PRIVILEG," Engl. "PRIVILEGE"; highHAP + NEU: e.g., "SATIRE," Engl. "SATIRE"; highHAP + POS: e.g., "EKSTASE," Engl. "ECSTASY") showed uncorrelated happiness and valence scores $(r=.09)$, indicating that lowHAP + POS words are perceived as being positive but not related to the discrete emotion happiness, that highHAP + NEU words are related to happiness but are not perceived as being positive, and so on. For statistical details about the stimulus set, see Table 1. Mean levels of arousal, imageability, (log-)frequency per million, bigram frequency, orthographic neighborhood size, frequency of orthographic neighbors, frequency of higher-frequent orthographic neighbors, and the mean numbers of letters, syllables, phonemes, and higherfrequency orthographic neighbors were controlled using analyses of variance (ANOVAs, all $F_{\mathbf{s}}<1$; see Table 1). Moreover, all control variables were matched for the highHAP-versuslowHAP and NEU-versus-POS contrasts, as we verified by means of pairwise $t$ tests (all $t \mathrm{~s}<1$ ). A list containing all words can be found in the supplementary materials. The 120 pronounceable but meaningless nonwords, matched to the words on numbers of letters and syllables $(t \mathrm{~s}<1)$, were taken from Briesemeister et al. (2014) as well. In addition, 30 filler items in the form of five pound signs (“\#\#\#\#”) were included, which was meant to increase the signal-to-noise ratio of the fMRI paradigm.

\section{Procedure}

While inside the scanner, participants received written instructions to decide as quickly and accurately as possible via buttonpress whether they were presented with a correct German word (index finger) or a nonword (middle finger). Moreover, they were instructed not to press any button when presented with fillers. Ten practice trials (four words, four nonwords, and two fillers) that were not part of the stimulus set described above were used to familiarize the participants with the task prior to the actual experiment.

The stimuli were presented in an event-related design via goggles using the Presentation software (Neurobehavioral Systems, Inc.), which also recorded response times and accuracy data. Each trial began with the presentation of a fixation cross $(+)$ in the center of the screen, which was presented for $2,500 \mathrm{~ms}$ on average (jitter: $2,000-3,000 \mathrm{~ms}$ ), followed by the stimulus $(1,500 \mathrm{~ms})$ at the exact same position. The stimuli were fully randomized without constraints for each participant individually and presented in white uppercase Arial letters on a black background, font size 50. Responses were given through a button box held in the right hand. The start of the first trial was controlled by an external pulse from the scanner. Finally, a 5-min anatomical $\mathrm{T} 1 \mathrm{scan}$ was recorded after completion of the lexical decision task.

\section{MRI data acquisition}

Neuroimaging was performed at the Dahlem Institute for the Neuroimaging of Emotion using a 3-T Siemens (Erlangen, Germany) Trim Trio MRI scanner equipped with a 12-channel head coil. Earplugs and headphones were used to attenuate the scanner noise, and form-fitting cushions were meant to prevent the participants' head movements. Functional imaging was done in a single run with 545 whole-brain T2*-weighted echoplanar images (EPI), recorded in ascending interleaved order (TR: 2,000 ms, TE: $30 \mathrm{~ms}, 70^{\circ}$ flip angle [FA], 37 slices, matrix: $64 \times 64$, field of view [FOV]: $192 \mathrm{~mm}, 3 \times 3$ $\times 3 \mathrm{~mm}$ voxel size, no gap). High-resolution T1*-weighted anatomic reference images were acquired as a set of 176 continuous sagittal slices (TR: 1,900 ms, TE: $2.52 \mathrm{~ms}, 9^{\circ}$ FA, matrix: $256 \times 256$, FOV: $256 \mathrm{~mm}, 1 \times 1 \times 1 \mathrm{~mm}$ voxels).

\section{Data preparation}

Mean lexical decision response times (LDRTs) were calculated for each condition and participant after the exclusion of nonresponders, behavioral errors, and outliers, which were defined as responses faster than $300 \mathrm{~ms}$ or slower than 1,500 ms. Error rates (ERRs) were calculated as the summed errors per condition and participant. Statistical analyses were computed using ANOVAs, as implemented in SPSS 13.0 (SPSS Inc., USA) at an a priori significance level of .05.

The neuroimaging raw data were preprocessed and analyzed using SPM 8 (available at www.fil.ion.ucl.ac.uk/spm/, accessed February 27, 2012). The images were slice-time corrected, realigned to the mean volume, unwarped, and 
normalized to the standard EPI template provided by the Montreal Neurological Institute (MNI) with $3 \times 3$ $\times 3 \mathrm{~mm}$ voxel sizes, and then smoothed with an 8 -mm (full width at half maximum [FWHM]) Gaussian kernel. For the statistical analyses, an event-related general linear model (GLM) analysis time-locked to the stimulus onset was used. On the first level, seven predictors were included as regressors in the design and were convolved with the canonical hemodynamic response function: the four word categories lowHAP + NEU, lowHAP + POS, highHAP + NEU, and highHAP + POS words, as well as nonwords, fillers, and trials excluded from the behavioral analyses (i.e., nonresponders, errors, and outliers as defined above). ${ }^{1}$ On the second level, participants were treated as random effects and the four word categories were included in a 2 (happiness) $\times 2$ (positivity) flexible factorial ANOVA. Main effects of positivity and happiness were analyzed with $F$ tests to test for main effects and possible interactions. Follow-up one-tailed $t$ tests were used to estimate the direction of the effects. To correct for multiple comparisons, peak voxel $(p<.001)$ and cluster size $(k>17)$ thresholds were used, following an a priori Monte Carlo simulation procedure proposed by Slotnick, Moo, Segal, and Hart (2003). To estimate the appropriate cluster threshold, 10,000 simulations were run with the corrected $p$ value set at $p<.05$ in the whole-brain analyses with an FWHM of 12 . Only activation clusters that survived these thresholds $(p>.001, k$ $>17$ ) are reported.

\section{Results}

Behavioral results

A 2 (happiness) $\times 2$ (positivity) repeated measures ANOVA revealed a significant main effect of positivity that was driven by faster responses for NEU than for POS words (detailed descriptive statistics are presented in Table 1, and inferential statistics in Table 2). Moreover, the Happiness $\times$ Positivity interaction approached significance ( $p=.053$, see Table 2$)$. Planned pairwise comparisons between all conditions were calculated and revealed faster processing for NEU words than for POS words within both the lowHAP and highHAP conditions. In addition, highHAP words were processed faster than lowHAP words within the POS condition, but not for the NEU words. These results are also depicted in Fig. 1.

ERRs ( $4 \%$ behavioral errors within the word materials) were analyzed using a repeated measures ANOVA comprising

\footnotetext{
${ }^{1}$ Inclusion of movement parameters as regressors caused spurious activations within the liquor, which is why they were not included in the model reported here. All significant effects within the a priori critical regions were robust against statistical checks for stimulus-related movement, however. Only the amygdala effect was less focused, expanding also to the hippocampus and the medial globus pallidus.
}

the $2 \times 2$ within-subjects factors Happiness and Positivity. A main effect of positivity was observed, based on fewer errors for NEU than for POS words (see Table 2). No further effects reached significance. Given that ERRs follow a binominal distribution (correct/incorrect), however, therefore violating several fundamental assumptions (see Jaeger, 2008), an additional mixed-effect logistic regression was calculated using JMP Pro 11 (SAS Institute Inc., USA). When we added participants and items as random effects to the model, the fixed effects (positivity, happiness, and their interaction) did not explain any variance. Thus, the ERR effect for positivity found in the ANOVA is not interpreted here.

\section{Neuroimaging results}

A 2 (happiness) $\times 2$ (positivity) flexible factorial ANOVA on the neuroimaging data revealed significant main effects for both factors, but no significant interaction between them. The happiness manipulation showed significant activation differences within the right amygdala, the cerebellum, and the left middle occipital gyrus (BA 19). Follow-up one-tailed $t$ tests revealed that all of these activation differences were related to increased differences for lowHAP as compared to highHAP words. The effects and the corresponding effect sizes, plotted as percentages of signal change within the peak voxel, are depicted in Fig. 2 using the SPM toolbox rfxplot (Gläscher, 2009).

The positivity manipulation affected activity within the precuneus (BA 7), mostly the left medial frontal gyrus (BA 8), the right superior temporal gyrus (BA 41), and two regions within the left inferior frontal gyrus. Direct contrasts between NEU and POS words revealed increased activation differences for POS words within the medial frontal and inferior frontal gyri. The reverse contrast revealed activation differences within the superior temporal gyrus and the precuneus, which are depicted in Fig. 3. It should be noted that in the onetailed contrasts, the inferior frontal gyrus effect for POS words and the superior temporal gyrus effect for NEU words were significant in both hemispheres.

\section{Discussion}

In the present study, we examined the three-level emotion processing hierarchy proposed by Panksepp (2012) and its applicability to affective-word processing studies. Specifically, two hypotheses were being tested. On the basis of the neural reuse hypothesis (M. L. Anderson, 2010; Herbert et al., 2009) and the assumption that affectively conditioned stimuli like words access the secondary process level within Panksepp's (2012) theory, we expected emotion networks like the anterior and posterior cingulate cortex, the medial 
Fig. 1 Behavioral lexical decision performance Depicted are the mean lexical decision response times (LDRTs), in milliseconds, and the mean summed error rates per condition. Error bars indicate one standard deviation

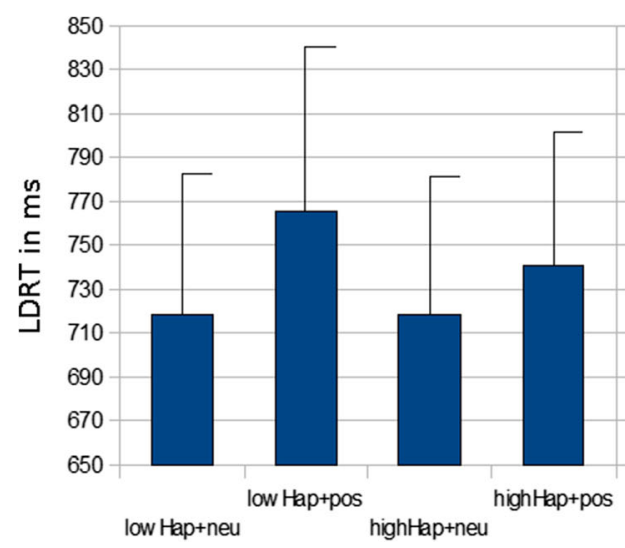

temporal lobe including hippocampus and parahippocampal gyrus, the amygdala, and the orbitofrontal cortex to be involved in affective word recognition (Citron, 2012; Herbert et al., 2009; Lewis et al., 2007; Nakic et al., 2006; Ponz et al., 2013; Schlochtermeier et al., 2013), despite the fact that the affective information was incidental to the task requirements (Kuchinke et al., 2005). In line with these predictions, and replicating results by Nakic et al. (2006), the amygdala was found to be engaged during implicit affective processing in the present study.

Fig. 2 fMRI results for the happiness contrast (highHAP vs. lowHAP). Depicted are structures that revealed significant differences for the happiness contrasts. Significant areas are labeled. The crossed lines in each panel indicate the voxel that was used to extract the mean activation depicted in the bar chart
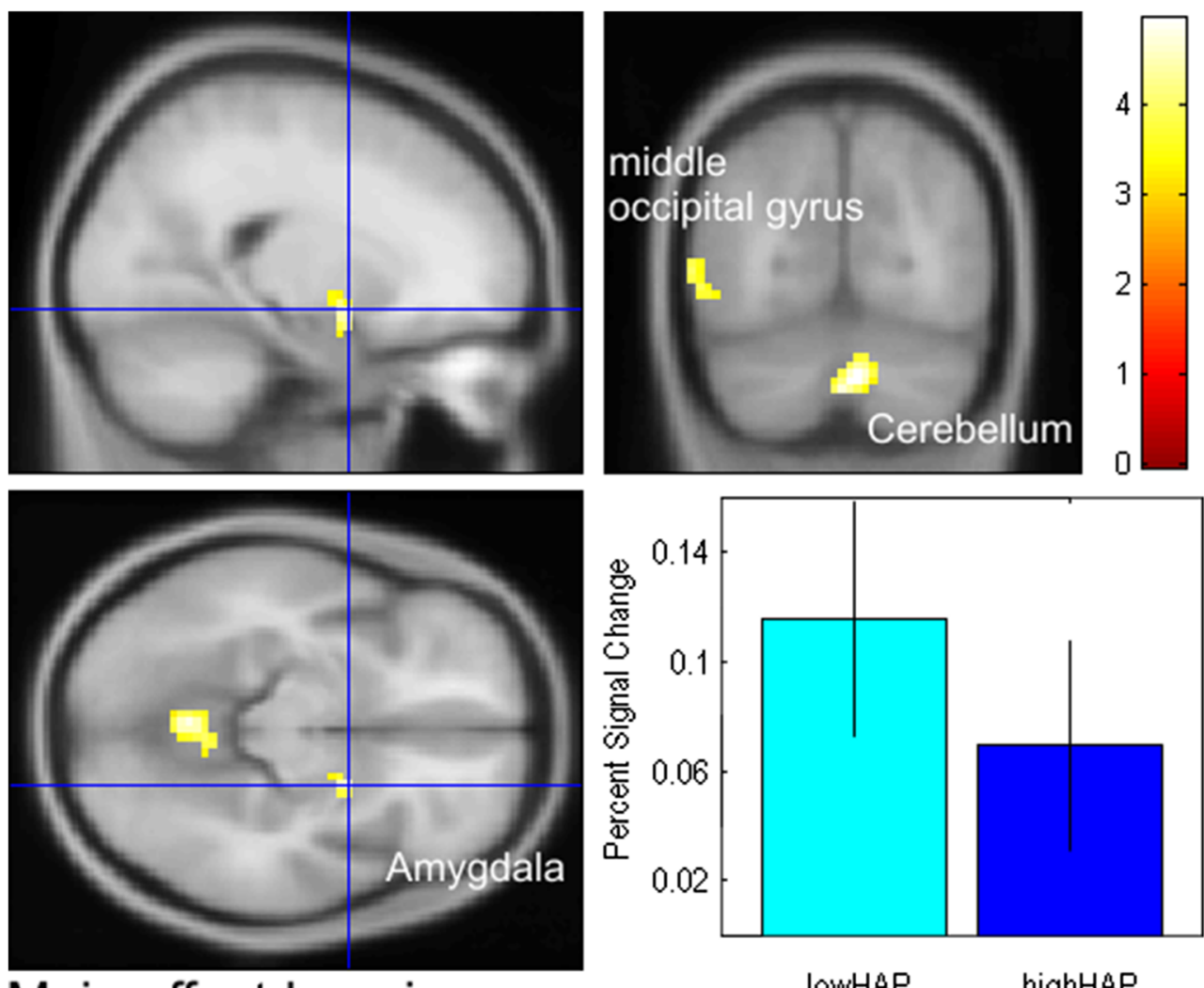

Main effect happiness
Referring to Kuchinke et al. (2005), Nakic et al. (2006) discussed amygdala activity during emotion word recognition in relation to behavioral performance. According to these authors, amygdala activation indicates emotional salience and serves as input for regions that are relevant for the behavioral response, such as the medial orbito-frontal gyrus and the anterior cingulate cortex, which in turn facilitate the behavioral lexical decision response. This interpretation is based on a significant correlation between amygdala and anterior cingulate cortex activity in conditions that show enhanced word-

lowHAP > highHAP 

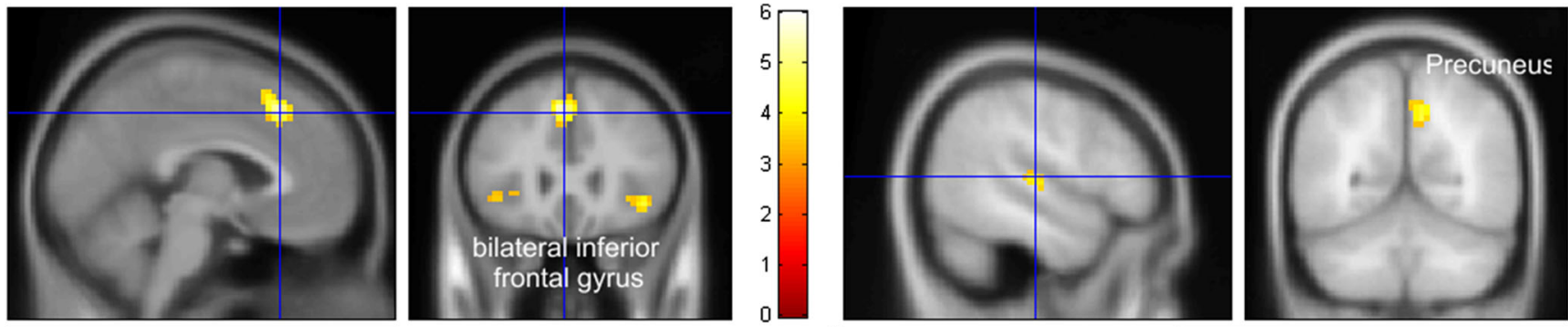

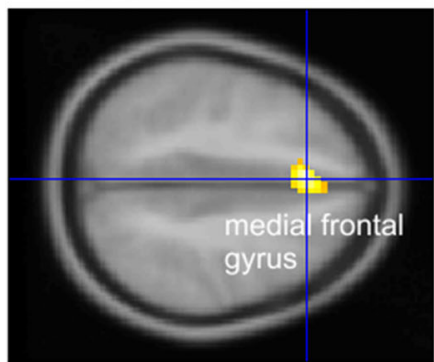

Main effect positivity

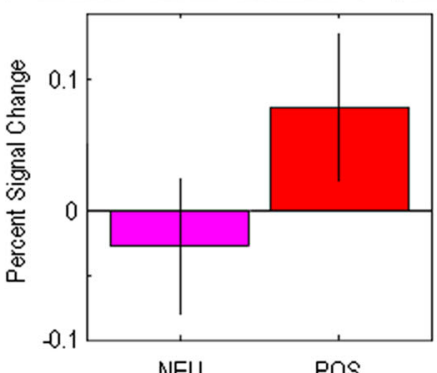

NEU

POS > NEU

Fig. 3 fMRI results for the positivity contrast (POS vs. NEU). Depicted are structures that revealed significant differences for the positivity contrasts, separated by direction. Significant areas are labeled. The crossed

processing speed - namely, for negative words in Nakic et al. (2006) - and the absence of comparable effects when no facilitated processing is observed (e.g., for negative words in Kuchinke et al., 2005). The present results additionally contribute to this discussion about the role of the amygdala in affective word processing: As was predicted by Nakic et al. (2006), affective information does not per se affect the amygdala, as indicated by the absence of activation differences for the positivity contrast. Instead, amygdala activation was observed for the happiness contrast, which showed no behavioral main effect on LDRTs or ERRs, which thus contradicts Nakic et al.'s (2006) assumptions. Given the theoretical framework of the present study, we interpret these results as suggesting that an explanation for activation differences within the amygdala, at least in the present study, must be related to the differentiation of discrete emotions versus affective dimensions.

Several recent studies have shown that manipulations along discrete emotion categories affect different word-processing variances than do manipulations along affective dimensions (Briesemeister et al., 2011a, 2011b, 2014; Silva et al., 2012; Weigand et al., 2013), suggesting that implicit processing of happiness and positivity reveals dissociable underlying networks. The results of Briesemeister et al. (2014) already supported this hypothesis: An early N1 effect, discussed as indexing early attentional resource allocation to affectively conditioned word forms (Bayer et al., 2012), was found to separate high- from low-happiness words, whereas positivity affected the N400 and the LPC. Both of the latter components are associated with explicit affective evaluation. No

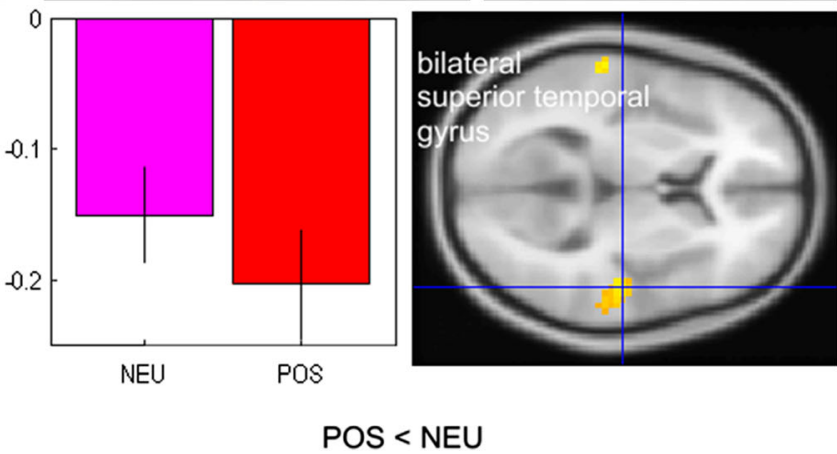

lines in each panel indicate the voxel that was used to extract the mean activation depicted in the bar chart

interactions were observed. ERP studies are an excellent way to investigate such temporal sequences, and the results directly supported the hierarchical emotion model, but they were not suited to specify the neuroanatomical networks at a fine-grained resolution. Panksepp (2012), however, made precise neuroanatomical predictions, which were tested and supported in the present study, by our finding of evidence for two nonoverlapping networks that underlie the processing of happiness- and positivity-related words. Activity differences were observed within the amygdala, the cerebellum, and the left middle occipital gyrus (see Table 2) when happiness words were processed. Most of these structures are part of Panksepp's (2012) secondary-process-level network, supposed to underlie the processing of conditioned PLAY (and thus, happiness) responses. Together with the happinessrelated N1 effect described in Briesemeister et al. (2014), these results support a fundamental role of discrete emotions during (implicit) affective processing. The positivity manipulation, in contrast, revealed differences within structures strongly associated within the so-called reading network underlying semantic processing - that is, within the superior temporal gyrus, the precuneus, and the medial and inferior frontal gyri (Binder \& Desai, 2011; Binder, Desai, Graves, \& Conant, 2009; Kuchinke et al., 2005). According to Panksepp, the tertiary process level "requires expansive neocortical tissues that permit lingustic-symbolic transformation" (Panksepp, 2005 , p. 32), which can explain activation differences related to semantic processing for positive words. Again, these results extend Briesemeister et al.'s (2014) finding of positivityrelated N400 and LPC effects, discussed as representing 
semantic evaluation and integration processes (e.g., Kutas \& Federmeier, 2011). Late ERP effects and neocortical reading network activity indicate that at least a minimum of lexicosemantic integration is about to happen when processing affective dimensions like positivity, given that secondaryprocess-level discrete-emotion differences are controlled. In line with this interpretation, in the present study we found positive words to be recognized significantly more slowly than neutral words, which is rather untypical (Briesemeister et al. 2012a, 2012b; Kuchinke et al., 2005) but replicates Briesemeister et al.'s (2014) data. We interpret this effect as an index of increased processing demands when affective properties (secondary process level) are controlled during implicit affect-based semantic categorization (tertiary process level), which is most pronounced in the lowHAP + POS condition (see Fig. 1).

Replicating Briesemeister et al.'s (2014) finding, again no interaction between happiness and positivity was observed in the neuroimaging data. Even though the power in the present study might have been too low to detect interactive effects, the absence of an interaction is well in line with previous reports of independent proportions of variance being explained by discrete-emotion and affective dimension manipulations (Briesemeister et al., 2011a, 2011b, 2014; Silva et al., 2012; Weigand et al., 2013), as is predicted by the hierarchical model (Panksepp, 2012). To the best of our knowledge, the present work is the first neuroimaging study that has tried to disentangle discrete-emotion and affective dimension influences in word recognition. Previous studies focused exclusively on the contribution of affective dimensions such as valence (Kuchinke et al., 2005; Maddock, Garrett, \& Buonocore, 2003; Nakic et al., 2006) and their interaction with arousal (Citron, Grey, Critchley, Weekes, \& Ferstl, 2014), revealing activation differences within affective as well as within semantic reading networks. Kuchinke et al. (2005), for example, found that positive words engage the superior frontal and orbito-frontal gyri and that negative words engage the inferior frontal gyrus, which was in line with and in the same direction as the present tertiary-process-level positivity effects in frontal regions. These results were interpreted as indexing explicit emotional memory functions. Additional activation differences when contrasting positive with neutral words were observed within the hippocampus, which is a secondaryprocess-level structure in Panksepp's theory and was interpreted as indexing memory-emotion interactions by Kuchinke et al. (2005). Citron et al. (2014) reported emotion-related activation differences during lexical decisions within the cerebellum and the parahippocampus, both of which are part of the secondary-process-level limbic pathways involved in cortical control of emotion. Further Valence $x$ Arousal interaction effects were found within the superior temporal gyrus, which relates to the present positivity results and which the authors interpreted as being responsible for the decoding of affective content from visual information (Citron et al., 2014). They, however, also noted that the superior temporal gyrus predominantly "is associated with semantic/ conceptual categorisation as well as comprehension of coherent, comprehensible text" (Citron et al., 2014, p. 87), two functions explicitly associated with the tertiary process level that was predicted to underlie the processing of positive words in the present study. Studies like these caused Panksepp (2012) to ask for a clearer distinction of the different affective processing levels in order to avoid causal misattributions, and the overall results presented here seem to support his view.

There were, however, also some unexpected effects. Both the present study and its predecessor (Briesemeister et al., 2014) showed clearly independent effects of positivity and happiness in the neurophysiological data, whereas on a behavioral level a Happiness $\times$ Positivity interaction was observed. The hierarchical emotion model is a neurophysiological model of emotion and motivation, which is why precise predictions concerning more subtle behavioral effects, especially in the context of visual word recognition tests, are outside its scope. Even most word recognition and reading models do not consider affective influences, although the extended multiple read-out model (MROMe) and the neurocognitive poetics models are notable exceptions (Kuchinke, 2007; Jacobs, 2011, 2014; see also Hofmann \& Jacobs 2014). The MROMe predicts that affective information will facilitate LDRTs at a prelexical level (see also Kissler \& Herbert, 2013, for first empirical evidence), although it does not make a distinction between discrete emotions and affective dimensions, and therefore cannot account for possible interactions. Moreover, the MROMe is not a neurophysiological model. Neural correlates are considered, but the focus is explicitly on the processes of visual word recognition. The present results suggest that a combination and integration of behavioral and neurophysiological data will be necessary to fully understand affective processing and visual word recognition alike, given that neurophysiologically separated structures can lead to interactive effects on the behavioral level. We assume that the different effect structures are caused by different time windows underlying behavioral and neurophysiological effects, but future theorizing should incorporate both levels of analysis to account for the existing complexities.

Despite their different foci, the MROMe and the hierarchical emotion model agree on the importance of the amygdala for affective processing (see also Siegle, Steinhauer, Thase, Stenger, \& Carter, 2002). It is well known that lesions within this structure can severely impair implicit affective processing (A. K. Anderson \& Phelps, 2001), but its functional role is still a matter of debate. Although the meta-analysis published by Sergerie, Chochol, and Armony (2008) focused on the amygdala's role in affective processing, highlighting its sensitivity to positive and negative stimuli alike, Pessoa and Adolphs 
(2010) argued that it is not affective content per se, but biological significance that engages the amygdala. In line with these hypotheses, previous affective-word processing studies have shown increased amygdala activity for both negative (e.g., Nakic et al., 2006) and positive (Schlochtermeier et al., 2013) words. The present data suggest an effect in the opposite direction, however - that is, increased right amygdala activation for lowHAP when compared with highHAP words (see Fig. 2). Whether this indicates that participants implicitly based their lexical decisions on highly emotional connotations of the stimulus material, which would make lowHAP words unexpected, and thus salient (Wright et al., 2001), can only be speculated. Given that the amygdala is known to engage in top-down influences to modulate (Pessoa \& Adolphs, 2010) and enhance the detection of task-relevant stimuli (Anderson \& Phelps, 2001), this could explain why no LDRT differences were found between lowHAP and highHAP words. Since Panksepp focused his research on primary-level-process emotions, he provided no detailed information on the amygdala's functional role. In any case, our finding of increased amygdala activity for lowHAP words is not predicted by the hierarchical emotion model.

It should also be noted that this work is based on positive word stimuli alone, which is a limiting factor in several ways. First of all, the hierarchical emotion model suggests seven primary-processlevel emotions in all (i.e., the positive emotions PLAY, LUST, CARE, SEEKING, and the negative emotions RAGE, PANIC, and FEAR). The present results so far relate to the PLAY system alone, but it would be interesting to see whether the three-level processing hierarchy, which is supposed to equally underlie all seven systems, applies for negative emotion words or for words related to a different positive primary emotional system (e.g., LUST), as well. Such a replication attempt could provide evidence for the generalizability of the present findings.

Second, and related to the focus on the positive end of the valence scale, it is possible that lowHAP and neutral words were not perceived as being neutral/lowHAP, but as being slightly negative relative to the positive/highHAP words. Recent research has shown that even everyday "neutral" objects can readily be categorized into positive and negative groups when strong affective anchors are absent (Bar \& Neta, 2007; Lebrecht, Bar, Barrett, \& Tarr, 2012). This suggests that the human brain calculates affective values not only depending on the presented stimulus, but also depending on earlier experiences and context (see also Barrett \& Bar, 2009; Westbury, Briesemeister, Hofmann \& Jacobs, 2014 under review). In the study by Bar and Neta, for example, they presented participants with different everyday neutral objects and showed stronger activation within the amygdala and precuneus for disliked sharp versus liked curved contours. Knowing that the amygdala activation observed for lowHAP words in the present study is discussed as being a crucial hub within the emotion-processing network sensitive to highly affective information (Siegle et al., 2002), and knowing that the precuneus activation observed for neutral words in the present study has previously been associated with selfreferential processing and episodic memory (Cavanna \& Trimble, 2006, mainly triggered by negative stimuli; see also Blood, Zatorre, Bermudez, \& Evans, 1999), the focus on positive emotions in the present study might have caused neutral/lowHAP words to be processed in brain regions associated with negative affect.

Third, Panksepp's (2012) theory is an emotion theory strongly focused on what he calls the "primary" process level, not a theory of affective word recognition. Nevertheless, several recent studies have suggested that emotional words are an appropriate material to test complex affective relationships (Briesemeister et al., 2011b, 2014; Silva et al., 2012; Weigand et al., 2013). We assume that affective words activate emotion-processing networks to varying degrees, and further that words being rated as strongly related to a given emotion activate the corresponding emotion networks with greater intensity than do words that are rated as weakly related to that emotion. Alternatively, it could also be the case that words strongly related to a given discrete emotion activate the underlying affective program in an "all-or-nothing" manner. The present design does not allow us to test between these two alternatives, and it is not clear which of the two mechanisms could be implemented on the secondary process level within the hierarchical model of Panksepp (2012). The model predicts, however, that the three-level hierarchy is independent of the stimulus material, which means that the predictions tested here would also apply to pictures, sounds, or any other emotionally laden stimuli. Keeping these limitations in mind, and awaiting further research, we propose that these first results provide initial support for Panksepp's (2012) assumption of a dissociable affective processing hierarchy, with happiness words engaging mostly secondary-process-level networks and positivity words relying on the semantic networks expected within the tertiary process level.

\section{References}

Anderson, A. K., \& Phelps, E. A. (2001). Lesions of the human amygdala impair enhanced perception of emotionally salient events. Letters to Nature, 411, 305-309.

Anderson, M. L. (2010). Neural reuse: A fundamental organizational principle of the brain. Behavioral and Brain Sciences, 33, 245266. disc. 266-313.

Bar, M., \& Neta, M. (2007). Visual elements of subjective preference modulate amygdale activation. Neuropsychologia, 45, 2191-2200.

Barrett, L. F., \& Bar, M. (2009). See it with feeling: Affective predictions during object perception. Philosophical Transactions of the Royal Society B, 364, 1325-1334. doi:10.1098/rstb.2008.0312

Bayer, M., Sommer, W., \& Schacht, A. (2012). P1 and beyond: Functional separation of multiple emotion effects in word recognition. Psychophysiology, 49, 959-969. 
Binder, J. R., \& Desai, R. H. (2011). The neurobiology of semantic memory. Trends in Cognitive Sciences, 15, 527-536.

Binder, J. R., Desai, R. H., Graves, W. W., \& Conant, L. L. (2009). Where is the semantic system? A critical review and meta-analysis of 120 functional neuroimaging studies. Cerebral Cortex, 19, 2767-2796. doi:10.1093/cercor/bhp055

Blood, A. J., Zatorre, R. J., Bermudez, P., \& Evans, A. C. (1999). Emotional responses to pleasant and unpleasant music correlate with activity in paralimbic brain regions. Nature Neuroscience, 2, 382-387.

Bohrn, I. C., Altmann, U., Lubrich, O., Menninghaus, W., \& Jacobs, A. M. (2013). When we like what we know-A parametric fMRI analysis of beauty and familiarity. Brain and Language, 124, 1-8.

Briesemeister, B. B., Hofmann, M. J., Kuchinke, L., \& Jacobs, A. M. (2012a). The BAWL databases in research on emotional word processing. In K.-M. Würzner \& E. Pohl (Eds.), Lexical resources in psycholinguistic research: Potsdam Cognitive Science Series (Vol. 3, pp. 61-66). Potsdam, Germany: Universitätsverlag.

Briesemeister, B. B., Kuchinke, L., \& Jacobs, A. M. (2011a). Discrete emotion norms for nouns: Berlin Affective Word List (DENNBAWL). Behavior Research Methods, 43, 441-448. doi:10.3758/ s13428-011-0059-y

Briesemeister, B. B., Kuchinke, L., \& Jacobs, A. M. (2011b). Discrete emotion effects on lexical decision response times. PLoS One, 6, e23743. doi:10.1371/journal.pone.0023743

Briesemeister, B. B., Kuchinke, L., \& Jacobs, A. M. (2012). Emotional valence-A bipolar continuum or two independent dimensions? SAGE Open October-December, 2, 2158244012466558

Briesemeister, B. B., Kuchinke, L., \& Jacobs, A. M. (2014). Emotion word recognition: Discrete information effects first, continuous later? Brain Research, 1564, 62-71. doi:10.1016/j.brainres.2014. 03.045

Cavanna, A. E., \& Trimble, M. R. (2006). The precuneus: A review of its functional anatomy and behavioural correlates. Brain, 129, 564 583. doi:10.1093/brain/aw1004

Citron, F. (2012). Neural correlates of written emotion word processing: A review of recent electrophysiological and hemodynamic neuroimaging studies. Brain and Language, 122, 211-226.

Citron, F. M. M., Grey, M. A., Critchley, H. D., Weekes, B. S., \& Ferstl, E. C. (2014). Emotional valence and arousal affect reading in an interactive way: Neuroimaging evidence for an approachwithdrawal framework. Neuropsychologia, 56, 79-89.

Duvarci, S., Popa, D., \& Paré, D. (2011). Central amygdala activity during fear conditioning. Journal of Neuroscience, 31, 289-294. doi:10.1523/JNEUROSCI. 4985-10.2011

Fritsch, N., \& Kuchinke, L. (2013). Acquired affective associations induce emotion effects in word recognition: An ERP study. Brain \& Language, 124, 75-83.

Gläscher, J. (2009). Visualization of group inference data in functional neuroimaging. Neuroinformatics, 7, 73-82.

Herbert, C., Ethofer, T., Anders, S., Junghofer, M., Wildgruber, D., Grodd, W., \& Kissler, J. (2009). Amygdala activation during reading of emotional adjectives - An advantage for pleasant content. Social Cognitive and Affective Neuroscience, 4, 35-49.

Hofmann, M. J., \& Jacobs, A. M. (2014). Interactive activation and competition models and semantic context: From behavioral to brain data. Neuroscience \& Biobehavioral Reviews, 46(1), 85-104. doi: 10.1016/j.neubiorev.2014.06.011

Jacobs, A. M. (2011). Neurokognitive Poetik: Elemente eines Modells des literarischen Lesens [Neurocognitive poetics: Elements of a model of literary reading]. In R. Schrott \& A. M. Jacobs (Eds.), Gehirn und gedicht: Wie wir unsere wirklichkeiten konstruieren [Brain and poetry: How we construct our realities] (pp. 492-520). München, Germany: Hanser.

Jacobs, A. M. (2014). Towards a neurocognitive poetics model of literary reading. In R. Willems (Ed.), Towards a cognitive neuroscience of natural language use. Cambridge, UK: Cambridge University Press.

Jacobs, A. M., \& Schrott, R. (2013). Gehirn und Gedicht: Wie Wörter emotional wirklich werden [Brain and poem: How words become emotionally real]. Keynote lecture given at the 55th Annual Conference of Experimental Psychologists (TeaP), Vienna, Austria.

Jaeger, T. F. (2008). Categorical data analysis: Away from ANOVAs (transformation or not) and towards logit mixed models. Journal of Memory and Language, 59, 434-446. doi:10.1016/j.jml.2007.11. 007

Johnson, M. K., Raye, C. L., Mitchell, K. J., Touryan, S. R., Greene, E. J., \& Nolen-Hoeksema, S. (2006). Dissociating medial frontal and posterior cingulate activity during self-reflection. Social Cognitive and Affective Neuroscience, 1, 56-64. doi:10.1093/scan/ns1004

Kissler, J., \& Herbert, C. (2013). Emotion, etmnooi, or emitoon?-Faster lexical access to emotional than to neutral words during reading. Biological Psychology, 92, 464-479.

Kuchinke, L. (2007). Implicit and explicit recognition of emotionally valenced words (Unpublished doctoral dissertation). Freie Universität, Berlin. Retrieved from www.diss.fu-berlin.de/diss/ receive/FUDISS thesis 000000002848

Kuchinke, L., Jacobs, A. M., Grubich, C., Võ, M. L.-H., Conrad, M., \& Herrmann, M. (2005). Incidental effects of emotional valence in single word processing: An fMRI study. NeuroImage, 28, 1022 1032 .

Kutas, M., \& Federmeier, K. D. (2011). Thirty years and counting: Finding meaning in the N400 component of the event-related brain potential (ERP). Annual Review of Psychology, 62, 621-647. doi:10. 1146/annurev.psych.093008.131123

Lebrecht, S., Bar, M., Barrett, L. F., \& Tarr, M. J. (2012). Micro-valences: Perceiving affective valence in everyday objects. Frontiers in Psychology, 3, 107.

LeDoux, J. E. (2000). Emotion circuits in the brain. Annual Review of Neuroscience, 23, 155-184. doi:10.1146/annurev.neuro.23.1.155

Lewis, P. A., Critchley, H. D., Rotshtein, P., \& Dolan, R. J. (2007). Neural correlates of processing valence and arousal in affective words. Cerebral Cortex, 17, 742-748.

Lindquist, K. A., Wager, T. D., Kober, H., Bliss-Moreau, E., \& Barrett, L. F. (2012). The brain basis of emotion: A meta-analytic review. Behavioral and Brain Sciences, 35, 121-143.

Maddock, R. J., Garrett, A. S., \& Buonocore, M. H. (2003). Posterior cingulate cortex activation by emotional words: fMRI evidence from a valence decision task. Human Brain Mapping, 18, 30-41.

Maren, S., Phan, K. L., \& Liberzon, I. (2013). The contextual brain: Implications for fear conditioning, extinction and psychopathology. Nature Reviews Neuroscience, 14, 417-428.

Nakic, M., Smith, B. W., Busis, S., Vythilingam, M., \& Blair, R. J. R. (2006). The impact of affect and frequency on lexical decision: The role of the amygdala and inferior frontal cortex. NeuroImage, 31, $1752-1761$.

Panksepp, J. (1998). Affective neuroscience: The foundations of human and animal emotions. New York, NY: Oxford University Press.

Panksepp, J. (2001). The neuro-evolutionary cusp between emotions and cognitions. Implications for understanding consciousness and the emergence of a unified mind science. Evolution and Cognition, 7, 141-163.

Panksepp, J. (2005). Affective consciousness: Core emotional feelings in animals and humans. Consciousness and Cognition, 14, 30-80.

Panksepp, J. (2006). The core emotional systems of the mammalian brain: The fundamental substrates of human emotions. In J. Corrigall, H. Payne, \& H. Wilkinson (Eds.), About a boy: Working with the embodied mind in psychotherapy (pp. 14-32). Hove, UK: Routledge.

Panksepp, J. (2007). Neurolizing the psychology of affects: How appraisal-based constructivism and basic emotion theory can coexist. Perspectives on Psychological Science, 2, 281-296. 
Panksepp, J. (2012). What is an emotional feeling? Lessons about affective origins from cross-species neuroscience. Motivation and Emotion, 36, 4-15.

Pessoa, L., \& Adolphs, R. (2010). Emotion processing and the amygdala: From a "low road" to "many roads" of evaluating biological significance. Nature Reviews Neuroscience, 11, 773-782.

Phillips, R. G., \& LeDoux, J. E. (1992). Differential contribution of amygdala and hippocampus to cued and contextual fear conditioning. Behavioral Neuroscience, 106, 274-285. doi:10.1037/07357044.106.2.274

Ponz, A., Montant, M., Liegeois-Chauvel, C., Silva, C., Braun, M., Jacobs, A. M., \& Ziegler, J. C. (2013). Emotion processing in words: A test of the neural re-use hypothesis using surface and intracranial EEG. Social Cognitive and Affective Neuroscience, 9, 619-627. doi: $10.1093 / \mathrm{scan} / \mathrm{nst} 034$

Satpute, A. B., Wager, T. D., Cohen-Adad, J., Bianciardi, M., Choi, J.-K., Buhle, J. T., . . . Barrett, L. F. (2013). Identification of discrete functional subregions of the human periaqueductal gray. Proceedings of the National Academy of Sciences, 110, 17101-17106.

Schlochtermeier, L. H., Kuchinke, L., Pehrs, C., Urton, K., Kappelhoff, H., \& Jacobs, A. M. (2013). Emotional picture and word processing: An fMRI study on the effects of stimulus complexity. PLoS One, 8, e55619. doi:10.1371/journal.pone.0055619

Sergerie, K., Chochol, C., \& Armony, J. L. (2008). The role of the amygdala in emotional processing: A quantitative meta-analysis of functional neuroimaging studies. Neuroscience \& Biobehavioral Reviews, 32, 811-830.

Siegle, G. J., Steinhauer, S. R., Thase, M. E., Stenger, V. A., \& Carter, C. S. (2002). Can't shake that feeling: Event-related fMRI assessment of sustained amygdala activity in response to emotional information in depressed individuals. Biological Psychiatry, 51, 693-707.

Silva, C., Montant, M., Ponz, A., \& Ziegler, J. C. (2012). Emotions in reading: Disgust, empathy and the contextual learning hypothesis. Cognition, 125, 333-338.

Slotnick, S. D., Moo, L. R., Segal, J. B., \& Hart, J., Jr. (2003). Distinct prefrontal cortex activity associated with item memory and source memory for visual shapes. Cognitive Brain Research, 17, 75-82. doi:10.1016/S0926-6410(03)00082-X

Võ, M. L.-H., Conrad, M., Kuchinke, L., Urton, K., Hofmann, M. J., \& Jacobs, A. M. (2009). The Berlin Affective Word List Reloaded (BAWL-R). Behavior Research Methods, 41, 534-538. doi:10. 3758/BRM.41.2.534

Weigand, A., Grimm, S., Astalosch, A., Guo, J. S., Briesemeister, B. B., Lisanby, S. H., . . . Bajbouj, M. (2013). Lateralized effects of prefrontal repetitive transcranial magnetic stimulation on emotional working memory. Experimental Brain Research, 227, 43-52.

Wicker, B., Keysers, C., Plailly, J., Royet, J.-P., Gallese, V., \& Rizzolatti, G. (2003). Both of us disgusted in my insula: The common neural basis of seeing and feeling disgust. Neuron, 40, 655-664. doi:10. 1016/S0896-6273(03)00679-2

Wilson-Mendenhall, C. D., Barrett, L. F., \& Barsalou, L. W. (2013). Neural evidence that human emotions share core affective properties. Psychological Science, 24, 947-956.

Wright, C. I., Fischer, H., Whalen, P. J., McInerney, S. C., Shin, L. M., \& Rauch, S. L. (2001). Differential prefrontal cortex and amygdala habituation to repeatedly presented emotional stimuli. NeuroReport, 12, 379-383. 